\title{
A NEW CO SURVEY OF THE MONOCEROS OB1 REGION
}

\author{
R.J. OLIVER ${ }^{1}$, M.R.W. MASHEDER ${ }^{1}$ AND P. THADDDEUS ${ }^{2}$ \\ ${ }^{1}$ Dept. of Physics, University of Bristol \\ ${ }^{2}$ Center for Astrophysics
}

\section{Survey}

This survey in the $(J=1 \rightarrow 0)$ line of $\mathrm{CO}$ at $115 \mathrm{GHz}$ was conducted in 1993 and 1994 using the $1.2 \mathrm{~m}$ millimeter-wave telescope at the CfA (see Oliver et al. 1996 for more complete description). The survey area, $\ell=196.0^{\circ}$ to $\ell=206.5^{\circ}, b=-1.5^{\circ}$ to $b=+3.5^{\circ}$ was covered on a square grid in $\ell$ and $b$ with spacings of 3.75 (0.4 FWHM) to give uniform sensitivity. All the spectra were position switched against positions measured be free of $\mathrm{CO}$ to $0.05 \mathrm{~K}$. Each spectrum has 256 channels $0.25 \mathrm{MHz}$ wide, giving a resolution of $0.65 \mathrm{~km} \mathrm{~s}^{-1}$ over $166 \mathrm{~km} \mathrm{~s}^{-1}$. The channel to channel noise temperature in the original spectra was $0.24 \mathrm{~K}$ (RMS) which was reduced to $0.115 \mathrm{~K}$ per beam by smoothing to $10^{\prime}$.

\section{Results}

This survey shows much more molecular gas in the Mon OB1 region and more complicated spatial and kinematic structures than previously known. Most of the emission is concentrated in two distinct velocity ranges (see Figure 1). The strongest emission is from gas in the Local arm at velocities between -5 and $+10 \mathrm{~km} \mathrm{~s}^{-1}$; the weaker is at velocities from $+15 \mathrm{~km} \mathrm{~s}^{-1}$ at $\ell=196^{\circ}$ to $+35 \mathrm{~km} \mathrm{~s}^{-1}$ at $\ell=206.5^{\circ}$ and is from the Perseus arm.

The large-scale velocity structure is not, however, completely described by two spiral arms. Notable departures are: (a) weak inter-arm emission at $\ell>203^{\circ}$, (b) two clouds with velocities that put them at kinematic distances beyond the Perseus arm (clouds 33 and 34 in the Figure 1) and (c) emission between $\ell=197.5^{\circ}$ and $\ell=201^{\circ}$ at 'forbidden' negative velocities ranging from -5 to $-15 \mathrm{~km} \mathrm{~s}^{-1}$ (clouds 4 and 9 in the Figure 1), which cannot result from differential Galactic rotation in the third quadrant. 


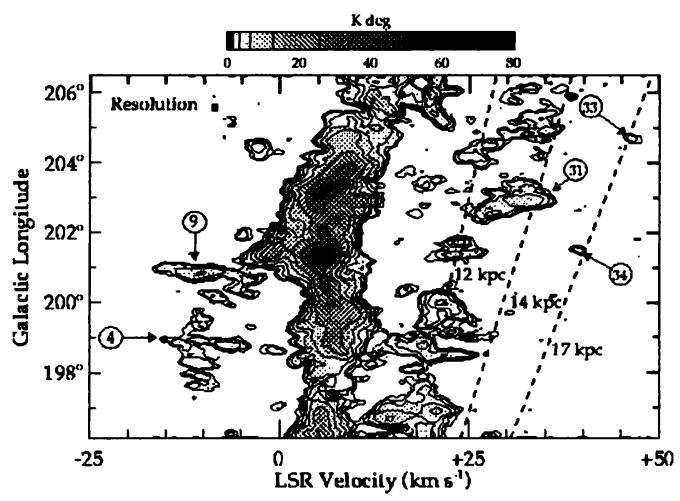

Figure 1. Longitude-velocity plot for CO detected toward the Mon OB1 region. The grey-scale ranges from $0.05 \mathrm{~K} \mathrm{deg}$ (white) to $74.0 \mathrm{~K} \mathrm{deg}$ (black). Contour levels are 0.05 , $0.1,0.2,0.4,0.8,1.6,3.2,6.4,12.8,25.6$, and $51.2 \mathrm{~K}$ deg.

The strongest $\mathrm{CO}$ emission $\left(\int T_{\mathrm{mb}} d v>16 \mathrm{~K} \mathrm{~km} \mathrm{~s}^{-1}\right)$ is well defined in discrete cores which are coincident with the young open clusters NGC 2245 , NGC 2247, NGC 2264 and other regions which have been independently identified as star forming. A striking result of this new survey is the large area in which weak $\mathrm{CO}$ emission $\left(\int T_{\mathrm{mb}} d v<5 \mathrm{~K} \mathrm{~km} \mathrm{~s}^{-1}\right)$ is now detected; $80 \%$ of the area surveyed has emission $>+3 \sigma, 60 \%$ of which is below the detection limit of Blitz's pioneering survey. This weak emission is spatially diffuse and fragmented, extending as halos around the strong emission previously reported towards the Cone Nebula and Mon R1

The strong $\mathrm{H}$ I emission of the Perseus arm has a weak molecular counterpart. The molecular clouds identified in this distant arm are comparable in mass and physical dimensions to an unbiased sample of local clouds. Moreover, the luminosity-linewidth relation for this unbiased sample of outer Galaxy clouds shows no significant deviation from that determined for the Brand and Wouterloot (1995) inner Galaxy cloud sample.

The association of Perseus arm clouds with colour-selected IRAS point sources provides evidence that they are forming massive stars and that the efficiency of such star formation is approximately independent of cloud mass. The discovery of these outer Galaxy clouds involved in massive star formation reaffirms the importance of studying the distribution of $\mathrm{CO}$ in the outer Galaxy.

\section{References}

Brand J., and Wouterloot J.G.A., 1995, Astron.Astrophys., 303, 851

Oliver R.J., Masheder M.R.W., Thaddeus P., 1996, Astron.Astrophys.315, 578. 